\title{
An ultra-narrow linewidth solution-processed organic laser
}

\author{
Oussama Mhibik ${ }^{1,2}$, Sebastien Forget ${ }^{1,2}$, Dan Ott $^{3}$, George Venus $^{3}$, Ivan Divliansky ${ }^{3}$, Leonid Glebov ${ }^{3}$ \\ and Sebastien Chénais ${ }^{1,2}$
}

Optically pumped lasers based on solution-processed thin-film gain media have recently emerged as low-cost, broadly tunable, and versatile active photonics components that can fit any substrate and are useful for, e.g., chemo- or biosensing or visible spectroscopy. Although single-mode operation has been demonstrated in various resonator architectures with a large variety of gain media-including dye-doped polymers, organic semiconductors, and, more recently, hybrid perovskites-the reported linewidths are typically on the order of a fraction of a nanometer or broader, i.e., the coherence lengths are no longer than a few millimeters, which does not enable high-resolution spectroscopy or coherent sensing. The linewidth is fundamentally constrained by the short photon cavity lifetime in the standard resonator geometries. We demonstrate here a novel structure for an organic thin-film solid-state laser that is based on a vertical external cavity, wherein a holographic volume Bragg grating ensures both spectral selection and output coupling in an otherwise very compact $\left(\sim \mathrm{cm}^{3}\right)$ design. Under short-pulse $(0.4 \mathrm{~ns})$ pumping, Fourier-transform-limited laser pulses are obtained, with a full width at half-maximum linewidth of $900 \mathrm{MHz}(1.25 \mathrm{pm})$. Using 20 -ns-long pump pulses, the linewidth can be further reduced to 200 $\mathrm{MHz}(0.26 \mathrm{pm})$, which is four times above the Fourier limit and corresponds to an unprecedented coherence length of $1 \mathrm{~m}$. The concept is potentially transferrable to any type of thin-film laser and can be ultimately made tunable; it also represents a very compact alternative to bulky grating systems in dye lasers.

Light: Science \& Applications (2016) 5, e16026; doi:10.1038/Isa.2016.26; published online 12 February 2016

Keywords: narrow linewidth; organic lasers; VECSEL; volume Bragg grating

\section{INTRODUCTION}

Lasers that are based on solution-processed thin-film gain materials ${ }^{1}$ have attracted interest for their wavelength agility across the whole visible spectrum, their compatibility with virtually all types of substrates, and their low-cost fabrication techniques. Lasing has been obtained in various gain media, including organic dye-doped polymer films or liquid crystals ${ }^{2}$, organic semiconductors ${ }^{3}$, colloidal quantum dots, and more recently hybrid perovskites ${ }^{4,5}$. Recent demonstrations of optical pumping of organic thin-film lasers by laser diodes and light-emitting diodes (LEDs) ${ }^{6-8}$ have confirmed the low cost and compactness potential of these technologies. Furthermore, organic and hybrid semiconductors can possibly be directly electrically pumped, although this possibility remains today an unsolved scientific challenge ${ }^{1}$.

Most of these laser devices can operate in a single-longitudinal mode (SLM), but no organic thin-film laser (or any solution-processed laser) has been demonstrated, until now and to the best of our knowledge, with a coherence length that is much longer than a few millimeters, or stated differently, with a linewidth that is well below a tenth of a nanometer. For applications such as holography, phase-sensitive sensing techniques, quantum information processing, or high-resolution spectroscopy; however, longer coherence lengths are desired. In this paper, we demonstrate an organic solid-state laser that is based on a rhodamine 640 dye-doped polymer film, in a Vertical External-Cavity Surface-emitting Organic Laser (VECSOL) ${ }^{9}$ in which the remote dielectric output coupler has been replaced by a volume Bragg grating $(\mathrm{VBG})^{10}$ (Figure 1). The coherence length is shown to be up to $1 \mathrm{~m}(200 \mathrm{MHz}$ or $0.26 \mathrm{pm}$ linewidth), which represents a linewidth reduction of two to three orders of magnitude compared to state-of-the-art thin-film lasers. The simple experimental arrangement, albeit not tunable in its present form, also enables a considerable gain in compacity and simplicity when compared to dye lasers, in which sub-GHz linewidths are obtained with grazing-incidence gratings ${ }^{11}$ or grating + prism combinations ${ }^{12}$. Hence, the lowest linewidth reported to date in a solid-state bulk dye laser is $350 \mathrm{MHz}$ in a dispersive long cavity ${ }^{13}$. A literature scan of reported linewidths in thinfilm laser architectures reveals that they all fall within the range of $\sim 0.01-10 \mathrm{~nm}$, with a vast majority standing at approximately $\sim 0.1$ $\mathrm{nm}$ (or $100 \mathrm{GHz}$ ). This finding holds true for distributed feedback (DFB) resonators, either the ones based on one-dimensional gratings ${ }^{14}$, two-dimensional photonic crystals ${ }^{15}$, or on single-transverse mode nanoribbon waveguides ${ }^{16}$, it is also valid for dye-doped bandedge DFB liquid crystal lasers ${ }^{2}$ as well as for Organic Vertical Cavity Surface Emitting lasers (OVCSELs or microcavities) ${ }^{17,18}$. 


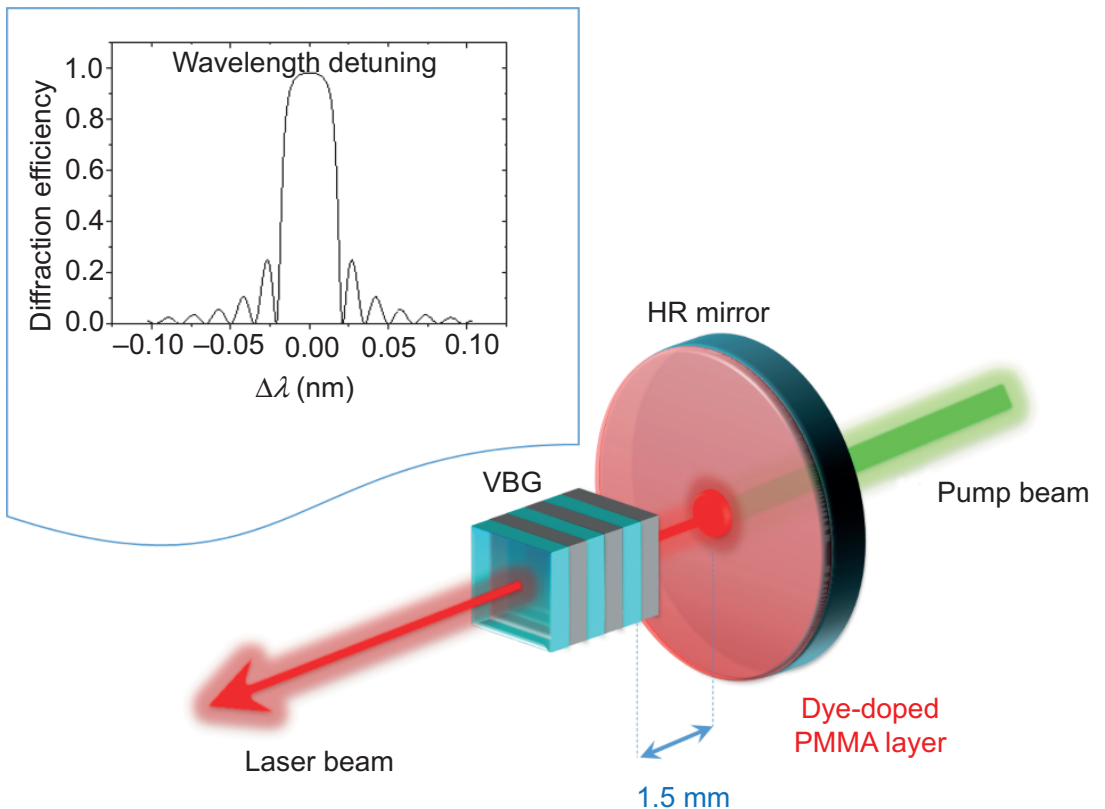

Figure 1 Schematic representation of the VBG-VECSOL setup. Inset: calculated diffraction efficiency at normal incidence vs. wavelength of the VBG used in this work. The central wavelength is $633.14 \mathrm{~nm}$.

\section{Design rules for ultra-narrow linewidth operation}

First to examine briefly the design rules for obtaining sub-pm (or $\mathrm{GHz}$ ) linewidth SLM operation and why such a regime is not attainable with standard thin-film laser architectures. A first design rule dictates that the photon cavity lifetime $\tau_{\text {cav }}=\left(2 \pi \Delta v_{c}\right)^{-1}$ ( $\Delta v_{c}$ is the cold cavity resonance linewidth) must be of the order of nanoseconds. The actual linewidth will be somewhat below $\Delta v_{o}$ but it gives a relevant order of magnitude, and this criterion also relates to the laser temporal dynamics under pulsed pumping. Assuming Fourier-transform (FT)limited pulses, the sub-GHz bandwidth requires a "smooth" spike-free pulse shape with a duration of $>\mathrm{ns}$, a condition that is met when $\tau_{\text {cav }}$ is matched with, or at least not too short compared to, the photoluminescence (PL) lifetime, which also falls in the nanosencond range for visible emitters.

The first condition is not met in OVCSELs because the cavity is too short $(<\mu \mathrm{m})$ : the finesses are typically $<10^{2-3}$, and $\tau_{\text {cav }}$ is less than 1 ps; hence, one of the lowest reported OVCSEL linewidths is $0.02 \mathrm{~nm}^{19}$ and corresponds to the Fourier limit of the first 100-ps relaxation pulse. In DFB planar resonators, although the propagation distances are longer, the condition is not achieved because scattering losses are high and lead to photon cavity lifetimes that are only a few hundred femtoseconds ${ }^{20,21}$, which is also associated with complex emission dynamics $^{20}$. Obtaining photon cavity lifetimes of up to the nanosecond level can be achieved in a high-finesse long $(>\mathrm{mm})$ external cavity, which is an architecture that is referred to as a VECSOL ${ }^{9}$. However, obtaining SLM lasing in a normal multimode long cavity will require very selective spectral filtering, which leads to the second design rule here: the intracavity spectral filter must have a bandwidth $\Delta v_{f}$ of the order of the free spectral range to secure the SLM operation. The reasons for such a stringent requirement originate in the ineffectiveness of mode competition in organic solid-state lasers. Unlike liquid continuous-wave dye lasers, for example, in which mode competition can be sufficiently effective to obtain SLM lasing even with poorly selective filters, in organic solid-state lasers, triplet state filling ${ }^{22}$ makes laser emission last only a few nanosenconds ${ }^{23}$ and up to at most several hundreds of nanoseconds $s^{7,8,24}$. This phenomenon is orders of magnitude shorter than the characteristic setting time $t_{c}$ that is required to reach SLM lasing in an ideal homogeneously broadened medium: ${ }^{25}$

$$
t_{c} \sim\left(\frac{\Delta v_{f, g}}{\mathrm{FSR}}\right)^{2} \tau_{\mathrm{cav}}
$$

where $\Delta v_{f, g}$ is the spectral filter or gain bandwidth. Indeed, for a 3-mmlong cavity, with a photon cavity lifetime of $\sim 1 \mathrm{~ns}$ and a $\sim 50 \mathrm{~nm}$ gain bandwidth that is typical of dyes, $t_{c}$ is orders of magnitude too long, in the millisecond range. It is then essential to incorporate a very selective spectral filter in the cavity, and for this purpose, a $\mathrm{VBG}^{10}$ is the ideal choice by virtue of its unique combination of high spectral and angular selectivity. The use of VBGs as output couplers or other components of laser resonators has enabled the demonstration of dramatic spectral and angular narrowing of emissions in semiconductor, solid-state and fiber lasers ${ }^{26}$ but has never been applied until now to organic and dye lasers. Moreover, VBGs recorded in photo-thermo-refractive (PTR) glass provide optical gratings that have a high diffraction efficiency, with also good thermal stability, a large transparency range of 350 $2700 \mathrm{~nm}$, and a high laser damage threshold (typically $>40 \mathrm{~J} \mathrm{~cm}^{-2}$ ) ${ }^{10}$.

\section{MATERIALS AND METHODS}

\section{VBG fabrication}

The recording medium for the VBGs used in these experiments is a $\mathrm{Na}_{2} \mathrm{O}-\mathrm{ZnO}-\mathrm{Al}_{2} \mathrm{O}_{3}-\mathrm{SiO}_{2}$ glass doped with silver, cerium, and fluorine, forming a PTR glass that is suitable for high-optical quality, low-loss phase hologram recording. The refractive index change is induced by a multi-step process as described by Glebov et al. ${ }^{27}$. The first step is the exposure of the glass sample to ultraviolet radiation in the range of 280$350 \mathrm{~nm}$. This exposure results in the photo-reduction of silver ions $\mathrm{Ag}^{+}$ to the atomic state $\mathrm{Ag}^{0}$. This stage is similar to the formation of a latent image in conventional photomaterials, and no significant changes in the optical properties of the PTR glass occur. The next step in the process is 
thermal development. A number of silver-containing clusters arise in the exposed regions of the glass after aging at elevated temperatures due to the increased mobility of $\mathrm{Ag}^{0}$ atoms. These silver-containing clusters serve as nucleation centers for $\mathrm{NaF}$ nanocrystal precipitation inside of the glass matrix. Interaction of those nanocrystals with the surrounding glass matrix causes a localized decrease in the refractive index.

Photo exposure of PTR to an interference pattern results in the recording of a periodic refractive index modulation that can be used as a volume phase grating. Reflecting Bragg gratings recorded in this fashion can be designed and characterized using Kogelnik's coupled wave theory ${ }^{28}$ and have maximum diffraction efficiency, exceeding $99 \%$. Here, we designed a reflective VBG with a long interaction length (the VBG length is $L_{\mathrm{VBG}}=8.3 \mathrm{~mm}$ ) and a small index of modulation $(\Delta n=63 \mathrm{ppm})$, to obtain a very narrow stopband $(\Delta \lambda=35 \mathrm{pm}$ fullwidth at half-maximum (FWHM), see inset of Figure 1) and a high diffraction efficiency $(\max . \mathrm{DE}=98 \%$ with $\pm 1 \%$ variations across the sample aperture). The central wavelength $(633.14 \mathrm{~nm})$ was chosen to fall into the emission spectrum of an efficient and stable red dye (Rhodamine 640) and slightly above the HeNe wavelength, to allow easy alignment. Given the small period of reflecting grating used in the experiments (approximately $200 \mathrm{~nm}$ ), an active stabilization technique was necessary to keep the grating's contrast high and prevent vibrations and instabilities in the recording system from washing out the grating pattern ${ }^{29}$. Because the total width between the first zeros is only $42 \mathrm{pm}$, SLM lasing is secured for cavity lengths of up to $4.8 \mathrm{~mm}$. Because the optical field is decaying inside the VBG, an effective length was estimated from reference ${ }^{30}$ to be $1.5 \mathrm{~mm}$ : we closed the cavity by a spherical mirror set at $1.5 \mathrm{~mm}$ away from the VBG facet to obtain an effective cavity length of $3 \mathrm{~mm}$.

\section{Description of the laser setup}

The setup (Figure 1) consists of a highly reflective dielectric spherical mirror $(R>99.5 \%$ within $600-660 \mathrm{~nm}$, transparent for the pump at $532 \mathrm{~nm}$, radius of curvature $R_{\mathrm{c}}=200 \mathrm{~mm}$ ) onto which an $18-\mu \mathrm{m}$-thick film of poly (methylmethacrylate) (PMMA) doped with $1 \mathrm{wt} \%$ of
Rhodamine 640 was spin-casted. The VBG closes the cavity and acts as the flat output coupler. Two different pump lasers were used in this study. In a first step, a frequency-doubled Nd:YAG laser (PowerChip from TeemPhotonics) emitting 0.4-ns FWHM pulses (at $10 \mathrm{~Hz}$ rep. rate) was used, which is hereafter referred to as a "short-pulse" pump laser. The maximum pump energy was $13 \mu \mathrm{J}$. In a second step, a "long-pulse" pump laser was used, which emitted 20-ns FWHM pulses with a maximum pulse energy of $140 \mu \mathrm{J}$. With these two lasers, the pump beam was focused to a $200-\mu \mathrm{m}$-in-diameter spot onto the active layer through the spherical mirror and ensured a good selective matching between the pump mode and the fundamental cavity mode, thus enabling a spatial single-mode operation.

The optical cavity has an overall volume of a few $\mathrm{cm}^{3}$ : we note that compactness, besides the obvious practical advantage, is also a critical requirement for a thin-film vertical cavity, due to the compulsorypulsed nature of the pump that is imposed by the triplet-state filling in organics: a short cavity enables the laser field to build up efficiently within the gain temporal window ${ }^{32}$.

\section{Optical characterization and linewidth measurement}

Laser energy was measured with a Spectrum detector Joulemeter (Delta DPM). The beam quality $\left(\mathrm{M}^{2}\right.$ factor) was measured from a measurement of the beam profile at different positions with a DataRay WinCam D beam profiler

The laser pulse intensity $I(t)$ was recorded with a high-speed photodiode (DET025A from Thorlabs, rise time $150 \mathrm{ps}$ ) that was attached to a 15-GHz (TDS6154C Tektronix) digital oscilloscope.

Laser spectra (see Figure 2) were recorded with a Jobin Yvon SPEX $270 \mathrm{M}$ with a resolution of $0.08 \mathrm{~nm}$.

The narrower linewidth of the VBG-VECSOL laser was then derived from a coherence length measurement performed with a Michelson interferometer, which is ideally suited for single-pulse measurements: the laser beam was collimated and split into two equally intense beams by a beam splitter and superimposed onto a charge-coupled device camera with a variable propagation time delay of $\tau$ (inset to Figure 3 ).

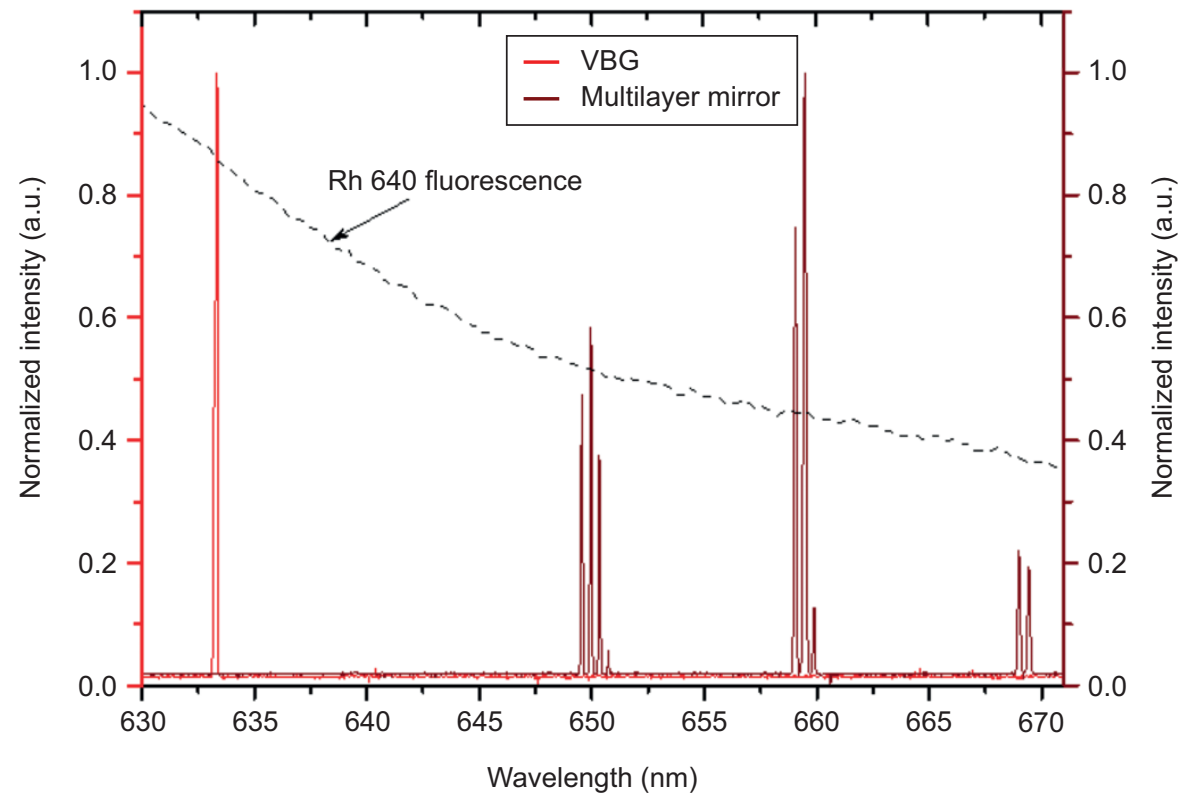

Figure 2 Laser spectra (recorded with 0.08-nm resolution spectrometer): VBG-VECSOL (left) with a 3-mm-long effective length and standard VECSOL (right) with a 0.5-mm cavity length, to resolve the cavity peaks (see text). The dashed curve represents the Rhodamine $640 \mathrm{PL}$ spectrum. The standard (free-running) VECSOL spectrum is red-shifted with respect to the PL maximum due to the overlap of the absorption and emission spectra at lower wavelengths. 
A fringe pattern (Supplementary Fig. S1) was obtained by making the two beams interfere with a small angle, which enabled the measurement of the visibility $V=\frac{I_{\max }-I_{\min }}{I_{\max }-I_{\min }}$ from the central fringes in one single image for a given optical path difference. Hence, the measured linewidth reflects the single-pulse linewidth, which is found to be stable from pulse to pulse (see Supplementary Fig. S1); however, because the cavity length is not stabilized, some of the spectral jitter arises from pulse to pulse. From the measured data, the numerical integration yields a coherence time that is defined by ${ }^{31}$ :

$$
\tau_{c}=\int_{-\infty}^{+\infty}\left|V^{2}(\tau)\right| d \tau
$$

\section{RESULTS AND DISCUSSION}

We first studied the laser characteristics under pumping by the "shortpulse" laser. The lasing spectrum recorded with the low-resolution grating spectrometer is shown in Figure 2. VBG lasing occurs as a single peak at the expected design wavelength of $633.14 \mathrm{~nm}$, with a measured linewidth that is equal to the spectrometer resolution $(0.08 \mathrm{~nm})$. To emphasize the ineffective mode competition in organic films, we reported in Figure 2 the emission spectrum that is obtained when the VBG is replaced by a dielectric mirror, which is set close to the spherical mirror $\left(L_{\mathrm{cav}}=0.5 \mathrm{~mm}\right.$ ), to enable the cavity modes to be resolved. Lasing is observed simultaneously with identical thresholds from three groups of peaks, in which each group corresponds to the transmission peaks of the etalon formed by the 18 - $\mu$ m-thick active layer.

The linewidth was inferred from a coherence time measurement that was performed with a Michelson interferometer, and the results are shown in Figure 3 (dots). From the variation in the visibility $V$ vs. the time delay, we found upon numerical integration (using Equation (2)) a coherence time of $\tau_{c}=0.7( \pm 0.05)$ ns, or a corresponding coherence length of $c \tau_{c}=20 \mathrm{~cm}$. Assuming a Gaussian's shape for the power density, the FWHM spectral width is $\Delta v=0.66 / \tau_{c}=930 \mathrm{MHz}(\Delta \lambda=1.25 \mathrm{pm})$ To infer whether the laser pulse is FT limited, $V(\tau)$ must be compared with the autocorrelation function of the field envelope ${ }^{31}$ :

$$
g(\tau)=\frac{\int \sqrt{I(t)} \sqrt{I(t+\tau)} d t}{\int I(t) d t}
$$

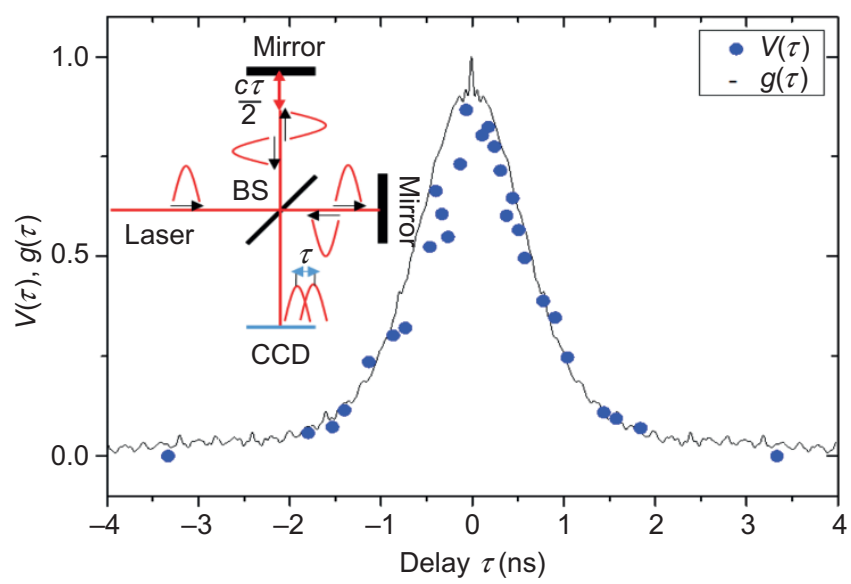

Figure 3 Comparison of the measured fringe visibility $V(\tau)$ with a Michelson interferometer (dots, simplified setup in inset) with the autocorrelation function $g(\tau)$ of the square root of the laser pulse intensity (solid line), for a VBG-VECSOL pumped with a 0.4-ns FWHM pump source. where $I(t)$ is the measured laser pulse intensity profile (see Supplementary Fig. S2), which has a width of 0.8 ns (FWHM). The function $g(\tau)$ is computed from $I(t)$, which neglects the instrumental response function. A good match between $V(\tau)$ (measured with a \pm $10 \%$ uncertainty) and $g(\tau)$ is apparent in Figure 3: the reduced $\chi^{2}$ factor that tests the goodness of curve matching ${ }^{33}$ is approximately 4 , which shows that the pulse is, within experimental uncertainty, FT limited.

To obtain a longer coherence length, we replaced the pump source by a "long-pulse" pump laser that emits 20-ns pulses. Under this regime, the laser pulse lasts approximately 15 ns (see inset of Figure 4 ). We extract from the measured visibility curve a coherence time $\tau_{c}=3.3$ ns, i.e., a coherence length of $1 \mathrm{~m}$.

Assuming a Gaussian shape for the power density, the FWHM spectral width is $\Delta v=200 \mathrm{MHz}(\Delta \lambda=0.26 \mathrm{pm})$. From the measured pulse shape (shown in the inset of Figure 4 ), the autocorrelation of the field envelope can be computed (Figure 4), and a decay time of $\tau_{d}=\int_{-\infty}^{+\infty}[g(\tau)]^{2} d \tau=13.5 \mathrm{~ns}$ is obtained, which means that the obtained spectrum is approximately four times broader than the Fourier Limit. Because the mechanical and acoustical noise are negligible in this range of frequencies, one possible reason for spectral broadening could originate in the small ripples that are apparent on the pulse laser shape, which reflect almost exactly the parasitic intensity modulations that are observed on the pump beam, in spite of an undetectable effect on the autocorrelation due to the low temporal resolution of the detection system. The laser performance is reported in Figure 5, with the 20-ns-long pump laser. The laser has a threshold

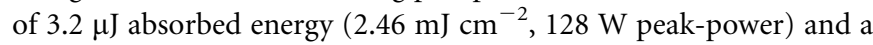
slope efficiency of $11 \%$. A comparable efficiency was obtained in a standard VECSOL in which the VBG was replaced by a dielectric coupler with $98 \%$ reflectivity. The output is linearly polarized, parallel to the pump polarization. A maximum pulse energy of $2.2 \mu \mathrm{J}$ was obtained, which corresponds to the peak power of approximately $140 \mathrm{~W}$. The Gaussian beam profile is shown in the inset of Figure 5 as well as the evolution of the beam radius at $1 / \mathrm{e}^{2} w(z)$ with a propagation distance, from which we derive a beam quality factor of $M^{2}=$ 1.09, which proves that lasing occurs on a single-transverse mode. The laser efficiency and linewidth is stable up to repetition rates of a few

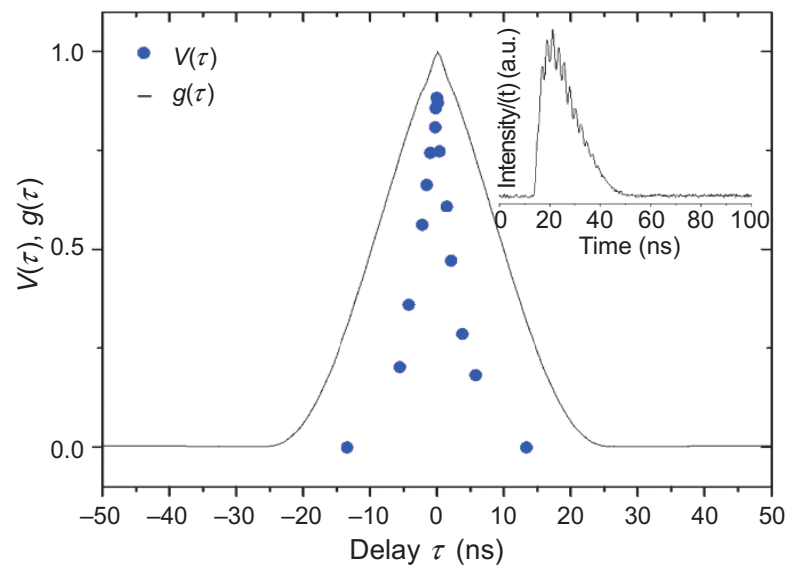

Figure 4 Long-pulse (20 ns) pump laser. Visibility $V(\tau)$ (dots) vs. time delay, measured from the central fringes of the interferograms (see Supplementary Fig. S1); calculated autocorrelation function $g(\tau)$ of the square root of $I(t)$ (solid line). The measured shape of $I(t)$ is shown in the inset. The coherence time deduced from $V(\tau)$ is $3.3 \mathrm{~ns}$, while the decay time of $g(\tau)$ is $13.5 \mathrm{~ns}$. 


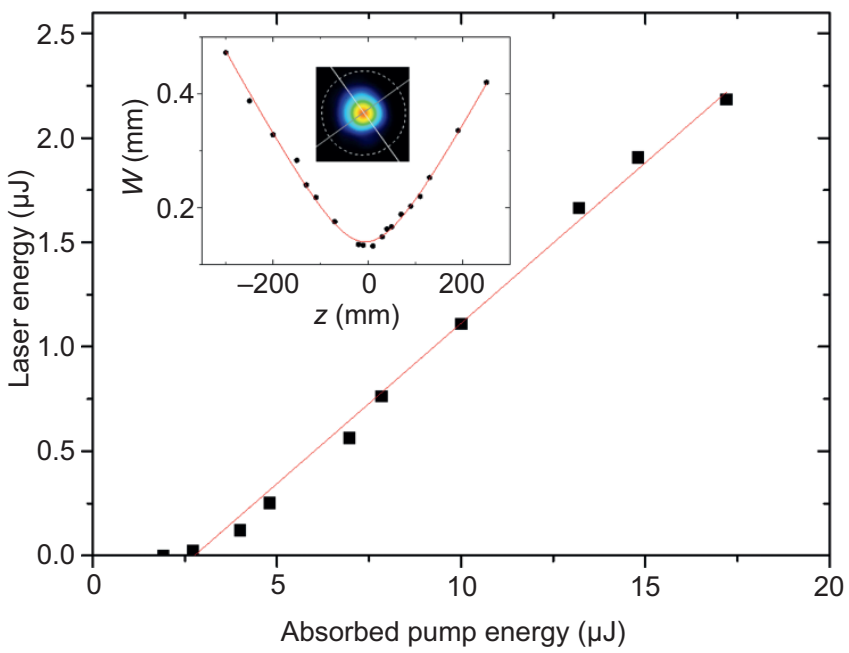

Figure 5 VBG-VECSOL efficiency under 20-ns-long pump pulses. The solid line is a guide for the eye. Inset: beam radius vs. propagation distance after a $400-\mathrm{mm}$ focal-length lens, used to derive a beam quality factor of $M^{2}=1.09$; photo: laser beam profile.

tens of $\mathrm{kHz}$, up to a point where photodegradation issues make the laser lifetime too short (a few seconds) to be practically operable.

The laser is similar to a standard multimode He-Ne laser (wavelength, coherence length, pure Gaussian mode, average power in the $\mathrm{mW}$ range when operated at $\mathrm{kHz}$ repetition rate) except that it emits bursts of hundreds of Watts, making it potentially useful in, e.g., dynamic holography.

\section{CONCLUSIONS}

In conclusion, we have shown that the spectral purity of a thin-film organic solution-processed solid-state laser can be enhanced by several orders of magnitude, down to $0.26 \mathrm{pm}$, due to the combination of a VBG with a vertical external cavity architecture. This association is a key enabler for photon cavity lifetimes that are in the nanosecond range, together with SLM operation, in contrast with the other more typical resonator structures that are used for organic thin-film lasers. Because continuous tunability is also possible using a chirped $\mathrm{VBG}^{34}$, this work opens new perspectives for developing compact, ultra-narrow linewidth, low-cost, and ultimately broadly tunable laser sources.

\section{ACKNOWLEDGMENTS}

The authors acknowledge the Agence Nationale de la Recherche (grant ANR12-EMMA-0040 "Vecspresso" project), the Region Ile de France (DIM Nano'K), and the LABEX SEAM for funding this work.

1 Chénais S, Forget S. Recent advances in solid-state organic lasers. Polym Int 2012 61: 390-406

2 Coles H, Morris S. Liquid-crystal lasers. Nat Photonics 2010; 4: 676-685.

3 Samuel IDW, Turnbull GA. Organic semiconductor lasers. Chem Rev 2007; 107: 1272-1295.

4 Xing GC, Mathews N, Lim SS, Yantara N, Liu XF et al. Low-temperature solutionprocessed wavelength-tunable perovskites for lasing. Nat Mater 2014; 13: 476-480.

5 Deschler F, Price M, Pathak S, Klintberg LE, Jarausch DD et al. High photoluminescence efficiency and optically pumped lasing in solution-processed mixed halide perovskite semiconductors. J Phys Chem Lett 2014; 5: 1421-1426.
6 Sakata $\mathrm{H}$, Takeuchi $\mathrm{H}$. Diode-pumped polymeric dye lasers operating at a pump power level of 10 mW. Appl Phys Lett 2008; 92: 113310.

7 Zhao Z, Mhibik O, Nafa M, Chénais S, Forget S. High brightness diode-pumped organic solid-state laser. Appl Phys Lett 2015; 106: 051112.

8 Yang Y, Turnbull GA, Samuel IDW. Hybrid optoelectronics: a polymer laser pumped by a nitride light-emitting diode. App/ Phys Lett 2008; 92: 163306.

9 Rabbani-Haghighi H, Forget S, Chénais S, Siove A. Highly efficient, diffractionlimited laser emission from a vertical external-cavity surface-emitting organic laser. Opt Lett 2010; 35: 1968-1970.

10 Efimov OM, Glebov LB, Glebova LN, Richardson KC, Smirnov VI. High-efficiency Bragg gratings in photothermorefractive glass. Appl Opt 1999; 38: 619-627.

11 Hänsch TW. Repetitively pulsed tunable dye laser for high resolution spectroscopy. Appl Opt 1972; 11: 895-898.

12 Costela A, García-Moreno I, Sastre R. Solid-state dye lasers. In: Duarte FJ, editor. Tunable Laser Applications. 2nd ed. New York: CRC Press; 2009.

13 Duarte FJ. Tunable organic dye lasers: physics and technology of high-performance liquid and solid-state narrow-linewidth oscillators. Prog Quantum Electron 2012; 36 29-50.

14 Voss T, Scheel D, Schade W. A microchip-laser-pumped DFB-polymer-dye laser. App/ Phys B 2001; 73: 105-109.

15 Baumann K, Stöferle T, Moll N, Raino G, Mahrt RF et al. Design and optical characterization of photonic crystal lasers with organic gain material. J Opt 2010; 12: 065003.

16 Deotare PB, Mahony TS, Bulović V. Ultracompact low-threshold organic laser. ACS Nano 2014; 8: 11080-11085.

17 Sudzius M, Langner M, Hintschich SI, Lyssenko VG, Fröb $\mathrm{H}$ et al. Multimode laser emission from laterally confined organic microcavities. Appl Phys Lett 2009; 94: 061102.

18 Yamashita K, Nakahata T, Hayakawa T, Sakurai Y, Yamao T et al. Vertical cavity surface emitting lasing from cyano-substituted thiophene/phenylene co-oligomer single crystals. Appl Phys Lett 2014; 104: 253301.

19 Bulović V, Kozlov VG, Khalfin VB, Forrest SR. Transform-limited, narrowlinewidth lasing action in organic semiconductor microcavities. Science 1998; 279: 553-555.

20 Zavelani-Rossi M, Perissinotto S, Lanzani G, Salerno M, Gigli G. Laser dynamics in organic distributed feedback lasers. App/ Phys Lett 2006; 89: 181105.

21 Vardeny ZV. Ultrafast Dynamics and Laser Action of Organic Semiconductors. New York: CRC Press; 2009.

22 Forget S, Chénais S. Organic Solid-State Lasers. Berlin: Springer-Verlag; 2013.

23 Schneider D, Rabe T, RiedI T, Dobbertin T, Kröger M et al. Laser threshold reduction in an all-spiro guest-host system. Appl Phys Lett 2004; 85: 1659-1661.

24 Zhang YF, Forrest SR. Existence of continuous-wave threshold for organic semiconductor lasers. Phys Rev B 2011; 84: 241301.

25 Jacquemet M, Domenech M, Lucas-Leclin G, Georges P, Dion J et al. Single-frequency $\mathrm{cW}$ vertical external cavity surface emitting semiconductor laser at $1003 \mathrm{~nm}$ and 501 $\mathrm{nm}$ by intracavity frequency doubling. Appl Phys B 2007; 86: 503-510.

26 Glebov L. High-performance solid-state and fiber lasers controlled by volume Bragg gratings. Rev Laser Eng 2013; 41: 684-690.

27 Glebov L. Photosensitive glass for phase hologram recording. Glastech Ber Sci Technol 1998; 71C: 85-90.

28 Kogelnik H. Coupled wave theory for thick hologram gratings. Bell Syst Tech J 1969; 48: 2909-2947.

29 Ott DB, Divliansky IB, Segall MA, Glebov LB. Stabilization system for holographic recording of volume Bragg gratings using a corner cube retroreflector. Appl Opt 2014; 53: 1039-1046.

30 Barmenkov YO, Dobryna Z, Salvador TP, Cruz JL, and Andrés MV. Effective length of short Fabry-Perot cavity formed by uniform fiber Bragg gratings Opt Express, 2006; 14: 6394-6399.

31 Saleh BEA, Teich MC. Fundamentals of Photonics. New York: John Wiley \& Sons, Inc.

32 Rabbani-Haghighi H, Forget S, Siove A, Chénais S. Analytical study of vertical external-cavity surface-emitting organic lasers. Eur Phys J Appl Phys 2011; 56: 34108.

33 Taylor JR. An Introduction to Error Analysis: The Study of Uncertainties in Physical Measurements. Sausalito: University Science Books; 1997.

34 Seger K, Jacobsson B, Pasiskevicius V, Laurell F. Tunable Yb: KYW laser using a transversely chirped volume Bragg grating. Opt Express 2009; 17: 2341-2347.

\footnotetext{
(c) (i) $\Theta$ This work is licensed under a Creative Commons Attribution-

NonCommercial-NoDerivs 4.0 Unported License. The images or other third party material in this article are included in the article's Creative Commons license, unless indicated otherwise in the credit line; if the material is not included under the Creative Commons license, users will need to obtain permission from the license holder to reproduce the material. To view a copy of this license, visit http://creativecommons.org/licenses/ by-nc-nd/4.0/
} 